\title{
Heart Track: Automated ECG Analysis for Detecting Myocardial Infarction
}

\author{
Varun S. Negandhi \\ K. J. Somaiya College \\ of Engineering.
}

\author{
Shruti D. Parab \\ K. J. Somaiya College \\ of Engineering.
}

\author{
Aishwarya A. \\ Walimbe \\ K. J. Somaiya College \\ of Engineering.
}

\author{
Poonam Bhogle \\ Assistant Professor \\ K. J. Somaiya College \\ of Engineering.
}

\begin{abstract}
The paper presents a system, Heart Track, which aims for automated ECG (Electrocardiogram) analysis. Different modules and algorithms which are proposed and used for implementing the system are discussed. The ECG is the recording of the electrical activity of the heart and represents the depolarization and repolarization of the heart muscle cells and the heart chambers. The electrical signals from the heart are measured non-invasively using skin electrodes and appropriate electronic measuring equipment. ECG is measured using 12 leads which are placed at specific positions on the body [2]. The required data is converted into ECG curve which possesses a characteristic pattern. Deflections from this normal ECG pattern can be used as a diagnostic tool in medicine in the detection of cardiac diseases. Diagnosis of large number of cardiac disorders can be predicted from the ECG waves wherein each component of the ECG wave is associated with one or the other disorder. This paper concentrates entirely on detection of Myocardial Infarction, hence only the related components (ST segment) of the ECG wave are analyzed.
\end{abstract}

\section{Keywords}

ECG, R-peak

\section{INTRODUCTION}

The proposed system concentrates on Myocardial Infarction whose analysis is done via the ECG wave. The ECG wave denotes the functioning of the human heart. Any change in the normal functioning of the heart has a direct impact on the structure of the ECG wave. The analysis of an ECG is done by the cardiologist who may or may not be available in all areas. The patient can use the system in such situations and if the machine shows the probability of getting a heart attack, he can go to the specialist. The system does not eliminate the need for the doctor but it acts as a second opinion to the doctor.

Myocardial Infarction occurs as a result of plaques (blockage) which are developed within the coronary arteries which obstruct the normal flow of blood and oxygen in the heart. If this process continues then it leads to heart attack. Myocardial means muscles of the heart and infarction is lack of oxygen.

Artery around the heart is surrounded by cardio myocytes(heart muscles) which are oddly connected. This allows them to pump the blood and oxygen to the heart efficiently. When plaques develop, the flow of blood decreases and so does the supply of oxygen to heart muscles.

The oxygen received by cardiomyocytes keeps on reducing and hence it slows down its rate of contraction. In order to compensate for the slow beating of this part of the heart, the rest of the heart beats faster than its normal speed. The membranes of cardiomyocytes begin to rupture. They then begin to inject a protein called troponin (protein found only in heart) into the bloodstream. A person begins to feel breathless at this point and feels fluid building inside his lungs. When the heart is not functioning properly, blood is accumulated into the heart and lungs as well. This back flow of blood makes breathing difficult. At this point the starving cardiomyocytes burst and die. If in such a condition, the patient is not treated within 20 minutes then his heart will not beat normally ever again because he is losing 500 heart cells per second. This condition overall is termed "Myocardial Infarction" [8].

The ECG wave depicts behaviour of heart under various disorders. Every segment of an ECG wave is associated with some disorder. The basic segments include the P wave, QRS complex, ST segment and T wave.

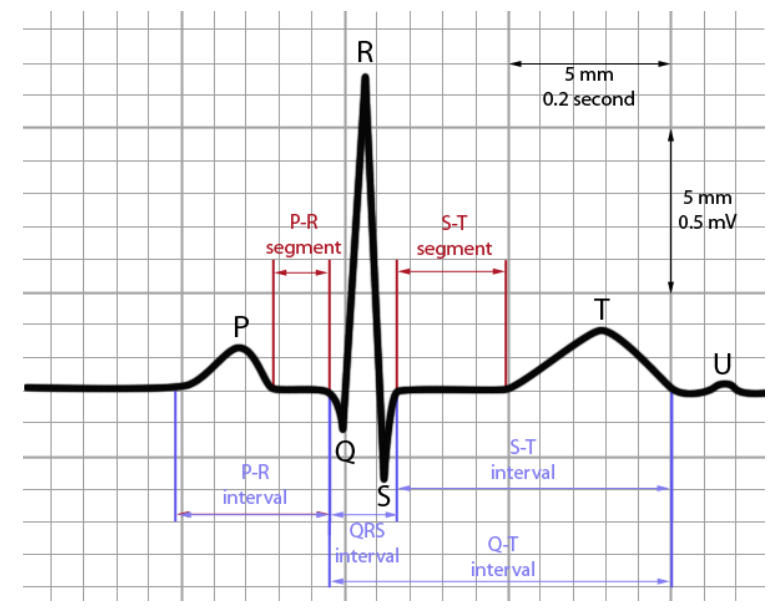

Figure 1: Typical ECG pattern [2].

This is the ECG wave for a normal heart whereas in case of Myocardial Infarction which deals with ST segment elevation, the ECG wave resembles the below pattern: 


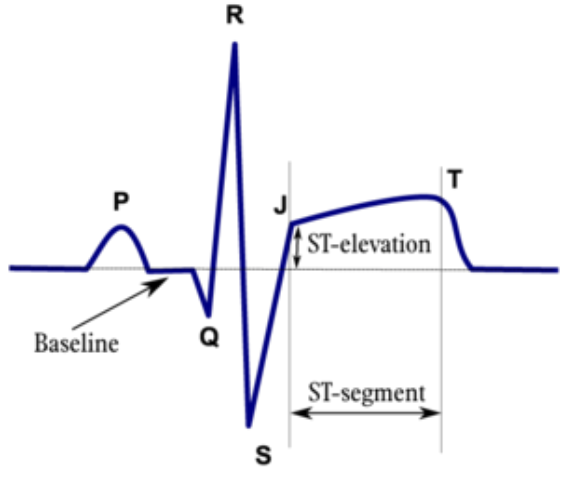

How to measure ST elevation?

Figure 2: ECG pattern with elevated ST segment in the presence of MI [9].

\section{PROPOSED SYSTEM}

Heart Track aims to provide automated analysis of digitalized ECG signals. This system concentrates on ECG wave feature extraction, localization of MI and culprit artery detection. The system accepts digitalized ECG signals as input, analyzes them with various algorithms and gives its diagnosis as output.

As the ECG pattern contains a large amount of information about the functionality of the heart as, for example its electrical conduction and also the amplitudes of the different waves ( $\mathrm{P}, \mathrm{QRS}$ and $\mathrm{T}$ wave) in a cardiac cycle, it can indicate an underlying heart disease.

This system is built in a manner such that it consists of modules such as ECG basic feature extraction like the R-peak to determine the exact window of the ECG cycle for the determination of the main segment known as the ST elevation. This detection of the ST elevation indicates the presence of MI. Further deductions concerning of exactly which part of the heart is at fault and the culprit artery leading to this effect are made from the localization algorithms.

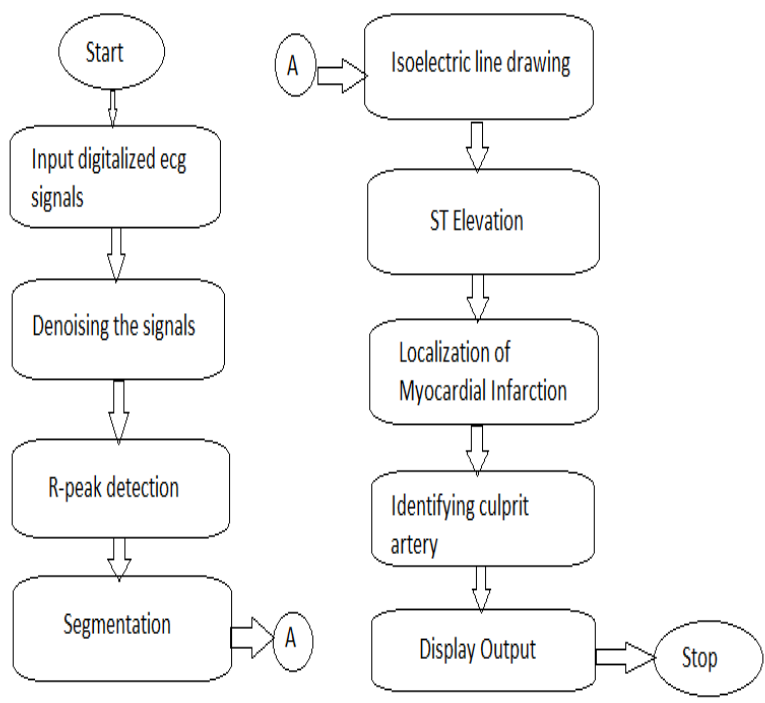

Figure 3: Architecture diagram for the proposed system.

The digitalized ECG signals are first fed into the module that uses Gaussian filter to smoothen the ECG waveform. It removes the deformities present in the signal to ensure accurate detection of various features of the waveform. The second module is to detect the R-peak of a sample. The ECG signal is a repeat of the cardiac cycle [7], so in order to decide on a single window of the cycle, the system does the $\mathrm{R}$ peak detection. Once the R-peak is detected the sample is segmented to decide on one particular instance of the waveform.

This segmented sample is then taken forward for further analysis. The next module plots the iso-electric line of the graph. This iso electric line is plotted to serve as the base line of reference for detecting ST elevation. The iso electric line is plotted using the algorithm that checks for the first elevation or depression in the signal. Once the iso-electric line is obtained, the next module is to identify the ST segment of the signal.ST segment is the part of the signal from the offset of QRS complex to the onset of the T-wave. In normal conditions, this ST segment coincides with the iso-electric line. The elevation of this segment with respect to iso-electric line is checked. This logic is used for all of the 12 ECG leads.

Once the ST elevation is detected, the localization is done on the basis of the presence of ST elevation in the leads. Each lead collects signals from a particular part of the heart [7]. Based on the presence of ST elevation, the location of the affected part of the heart is predicted. The further step is to determine the artery at fault. By comparing the presence of the ST elevation and also the extent of elevation, the artery which might contain blockage is predicted with the help of an algorithm for culprit artery detection. The above steps are repeated for all of the 12 leads of the ECG. This paper contains snapshots of only one of the 12 leads. The summarized results are then obtained as output to the user.

\section{SMOOTHENING}

Gaussian filter is used to remove noise from the signal using a convolution operator. This makes detection of R-peak easy. The Gaussian operator uses a kernel (smallest matrix used for convolution) which is a 'bell-shaped' curve.

The Gaussian distribution for 1-D signal [10]:

$$
G(\mu, \sigma, t)=\frac{1}{\sqrt{2 \pi \sigma^{2}}} e^{-\left\{\frac{(t-\mu)^{2}}{2 \sigma^{2}}\right\}}
$$

where, $\sigma$ is the standard deviation of the distribution and $\mu$ is the mean.

The degree of smoothness depends upon the value of standard deviation taken. Larger values of standard deviation require large kernels. The Gaussian filter brings the signal closer to the value of the central pixels and hence smoothened signal is obtained. There are various approaches for smoothening the signal. However, all those approaches do not provide the expected result. The following figures show the result of using these approaches on the original signal.

Two more wavelet transforms using $\mathrm{db} 4$ and sym 4 wavelet were tested on approximately 20-30 samples for smoothening the signals. It was found that the Gaussian filter yielded best results. The following snapshots show the implementation results. 


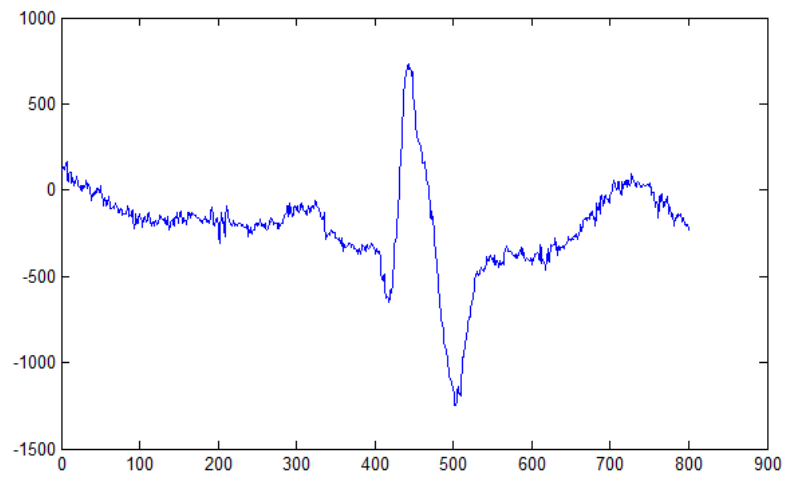

Figure 4: Original ECG waveform.

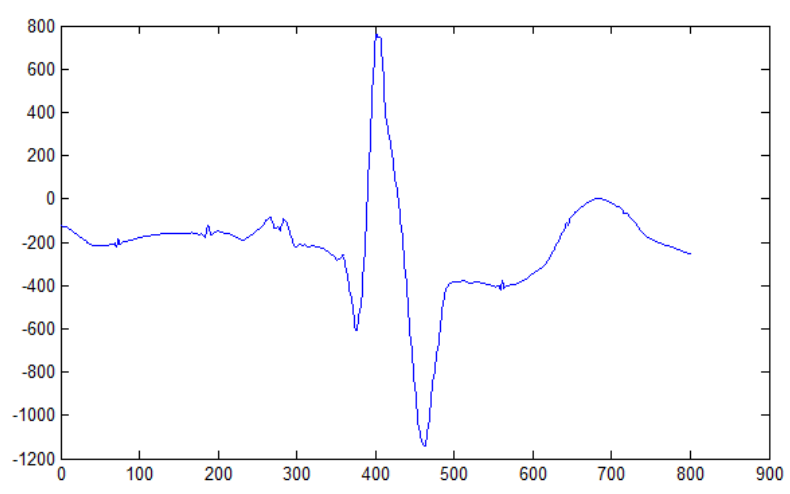

Figure 5: Smoothening using sym4 wavelet.

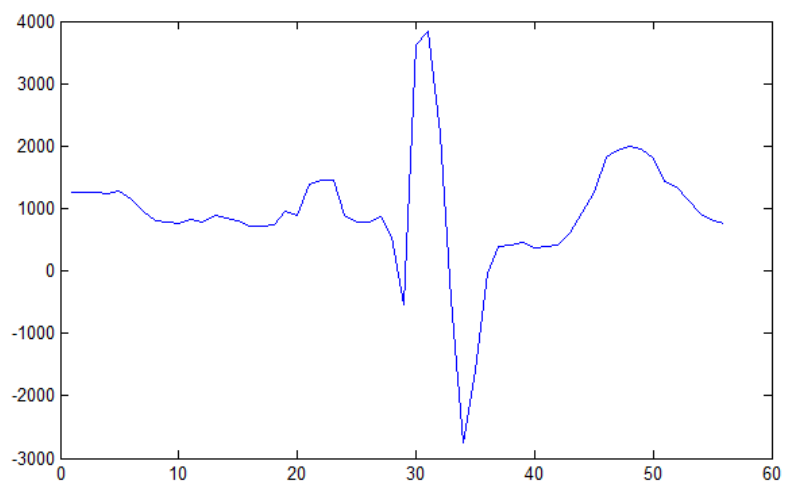

Figure 6: Smoothening using db4 wavelet.

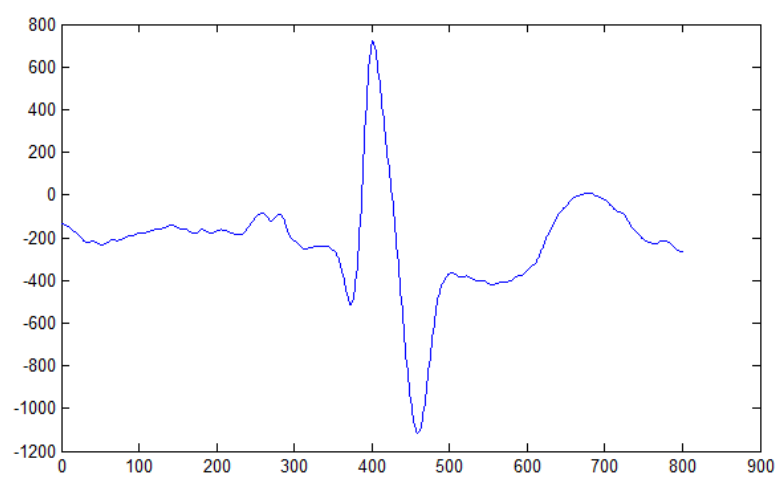

Figure 7: Smoothening using Gaussian filter.

The primary focus in the signal is detection of the ST elevation. The result obtained after smoothening from sym 4 and $\mathrm{db} 4$ wavelets still contain small peaks in the wave. These peaks create complications while detecting R-peak due to their sharpness. As seen in the last figure, Gaussian Filter gives a smooth signal which satisfies the required criteria.

\section{R-PEAK DETECTION}

The ECG signal comprises of a number of instances of the cardiac cycle. According to the medical practitioners it is observed that, the ECG signal typically repeats itself after 0.7 seconds []. For the purpose of diagnosis, the system concentrates only on one sample of the PQRST wave. In order to detect one sample, the R-peak is to be located and further, the corresponding sample is segmented from the signal.

R-peaks are fundamentally the sharpest peaks in the ECG waveforms. This characteristic feature is used for the detection of R-peaks. The algorithm starts with identifying the highest point in the signal. This point is checked for the sharpness by comparing the slopes of the adjacent points of the signal which are placed 0.005 seconds apart from the point. If the slope is steep enough, the point is concluded to be the R-peak; else the algorithm looks for the highest point in the next window of time period 0.01 seconds. This procedure continues till the algorithm finds an accurate R-peak.

\subsection{Algorithm for R-peak detection}

1. Find the highest point in the signal say max.

2. Select two points, 0.005 seconds to the left and 0.005 seconds to the right of the max.

3. Calculate the slope of these two points with respect to max.

4. If the slope of both the points is greater than or equal to 3, conclude that max is the R-peak.

5. Else, calculate the range of next window of time period 0.01 seconds. Find the highest point in this window which becomes the max and continue from step 2.

This algorithm was formulated and tested on approximately 10-20 samples obtained from the Physionet PTB Diagnostic ECG Database[5]. The values of the slope and the period of the window were determined after analyzing the results of the tested samples. The following table summarizes the results of the analysis:

Table 1: Results of analysis of testing on samples.

\begin{tabular}{|c|c|c|c|}
\hline $\begin{array}{c}\text { Slope limit } \\
\text { for } \\
\text { deciding } \\
\text { sharpness } \\
\text { of R-peak }\end{array}$ & $\begin{array}{l}\text { 5: could not } \\
\text { detect some } \\
\text { R-peaks } \\
\text { which are not } \\
\text { quite sharp. }\end{array}$ & $\begin{array}{l}\text { 2: considered } \\
\text { unwanted } \\
\text { peaks as R- } \\
\text { peaks. }\end{array}$ & $\begin{array}{c}\text { 3: detected } \\
\text { R-peaks in } \\
\text { most of the } \\
\text { cases. }\end{array}$ \\
\hline $\begin{array}{c}\text { Selection } \\
\text { of left and } \\
\text { right } \\
\text { adjacent } \\
\text { points } \\
\end{array}$ & $\begin{array}{l}\text { 0.01s: detects } \\
\text { unwanted } \\
\text { steep P or T } \\
\text { waves. }\end{array}$ & $\begin{array}{l}\text { 0.003s: cannot } \\
\text { detect R- } \\
\text { peaks with } \\
\text { slightly blunt } \\
\text { points. }\end{array}$ & $\begin{array}{l}0.005 \mathrm{~s}: \\
\text { detects R- } \\
\text { peaks in } \\
\text { most of the } \\
\text { cases. }\end{array}$ \\
\hline $\begin{array}{c}\text { Window } \\
\text { size }\end{array}$ & $\begin{array}{l}\text { 0.03s: very } \\
\text { thin, inverted } \\
\text { R-peaks } \\
\text { cannot be } \\
\text { detected. }\end{array}$ & $\begin{array}{c}0.01 \mathrm{~s}: \\
\text { detected R- } \\
\text { peaks in most } \\
\text { of the cases. }\end{array}$ & \\
\hline
\end{tabular}

The values as illustrated above are a result of testing on ECG samples. Out of these, the best values for slope limit of Rpeak, selection of left and right adjacent points and the window size are $3,0.005 \mathrm{~s}$ and $0.01 \mathrm{~s}$ respectively. Hence the 
use of these values in the above mentioned algorithm is justified.
Following are the some implementation results where the $\mathrm{R}$ peaks are accurately detected for different waveforms.

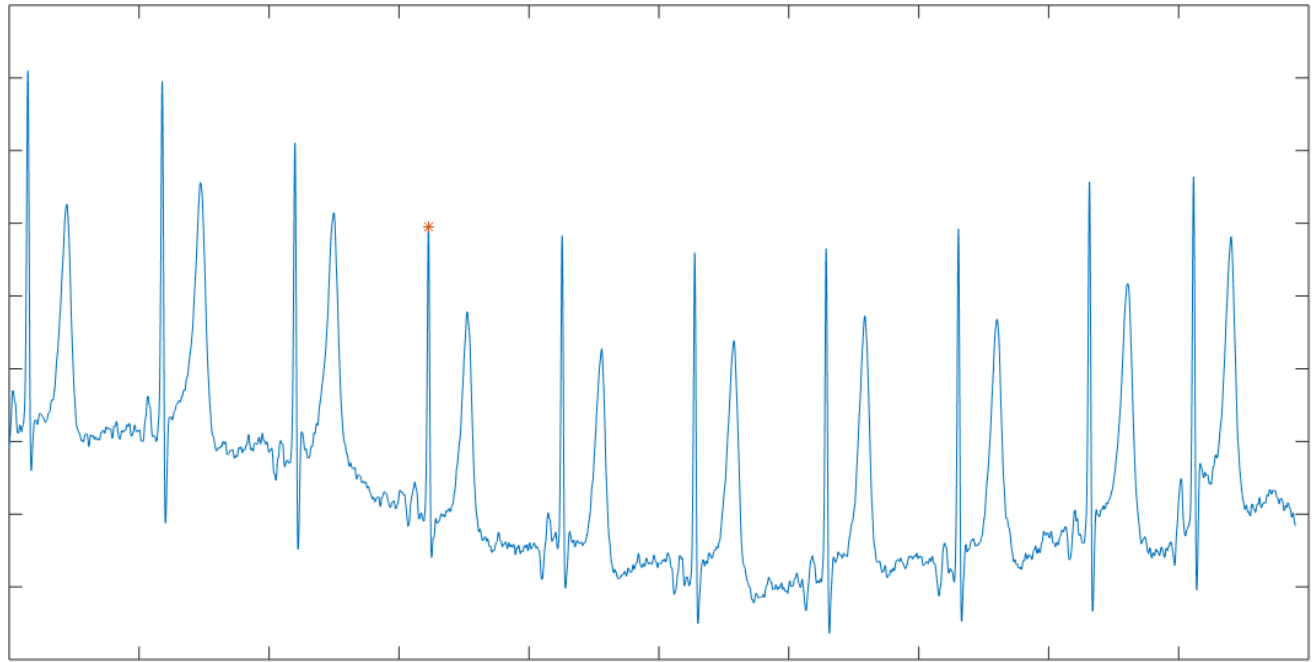

Figure 8: Accurate $\mathbf{R}$ peak detection for one of the 12 leads.

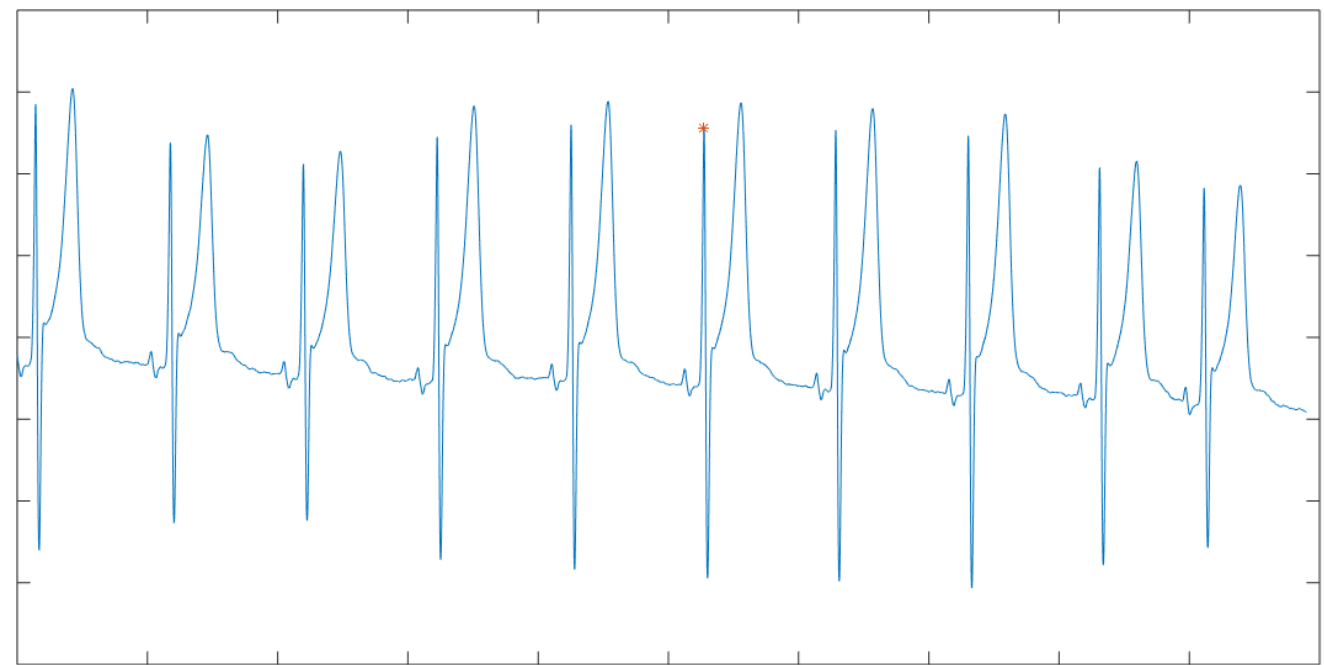

Figure 9: Accurate $\mathbf{R}$ peak detection for one of the 12 leads.

\section{ISO-ELECTRIC LINE DETERMINATION}

The iso-electric line serves as the base line of reference for detecting ST elevation. In normal conditions, this ST segment coincides with the iso-electric line. The elevation of this segment with respect to iso-electric line is checked.

This elevation of the ST segment predicts the presence of Myocardial Infarction. Thus, plotting the line of reference is the basis for the further deductions.

After the R peak is detected, the signal is segmented again and a single cardiac cycle is considered for further analysis. This algorithm checks for the first elevation or depression in the signal. Every cardiac cycle starts with either an elevation or depression which is known as the $\mathrm{P}$ wave. Thus, considering this elevation/depression as the onset of the wave, the isoelectric line is plotted .Once the iso-electric line is plotted the ST segment is located and whether or not the elevation is present is identified.

\subsection{Algorithm for detection of iso-electric} line

1. Consider a segmented signal obtained after the R-peak detection and calculate its length.

2. Now traverse this sample along its $\mathrm{x}$-axis to determine the onset of the signal. This onset might be an elevation or depression i.e. it's either a $\mathrm{P}$ wave or an inverted $\mathrm{P}$ wave respectively.

3. Now traversing along its length, calculate the distance between every corresponding y co-ordinates.

4. Considering this distance as a difference, it can be considered as an elevation if the difference is positive or depression when it's negative.

5. Mark its corresponding initial x-co-ordinate and plot the line of reference along the signal.

The above algorithm was tested on a set of 20-30 samples. 


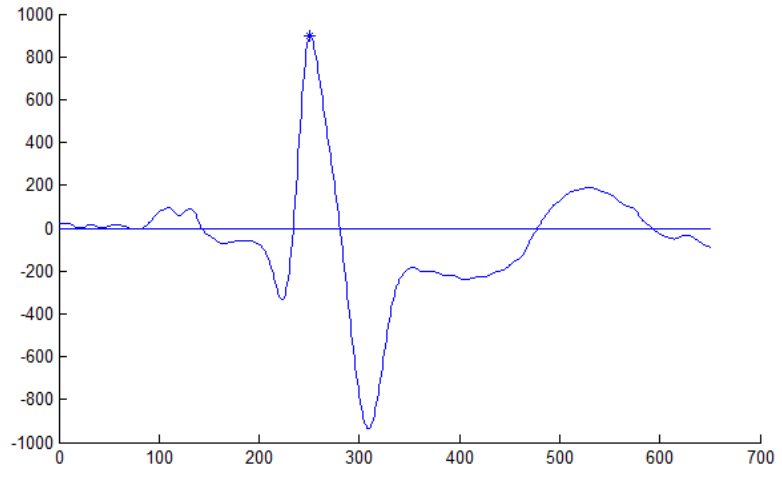

Fig 10: Implementation result of iso-electric line detection.

The above snapshot represents the implementation results.

\section{ST ELEVATION DETECTION}

The segment between the offset of $\mathrm{S}$ wave and the onset of $\mathrm{T}$ wave is known as ST segment. This is the most critical module of the system. The occurrence of an ST elevation in an ECG graph denotes whether a person is suffering from MI.

With respect to ST elevation, there exists a dichotomy in the ECG graph pattern. This is with respect to the position of S point, either below the iso-electric line or not. This scenario is explained in detail in the following two cases:

\subsection{Case 1:}

This case considers the scenario where the $\mathrm{S}$ point is below the iso-electric line. In such a case, the algorithm traverses towards the right to find the intersection point with the isoelectric line and then determines the ST segment elevation.

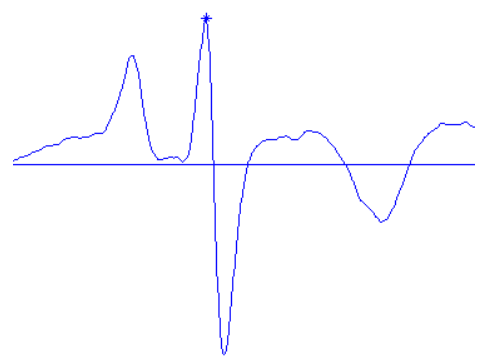

Figure 11: A sample where the $S$ point is below the isoelectric line.

\subsection{Case 2:}

In this case, the $\mathrm{S}$ point is not below the iso-electric line. Hence, the algorithm directly concludes the presence of ST elevation.

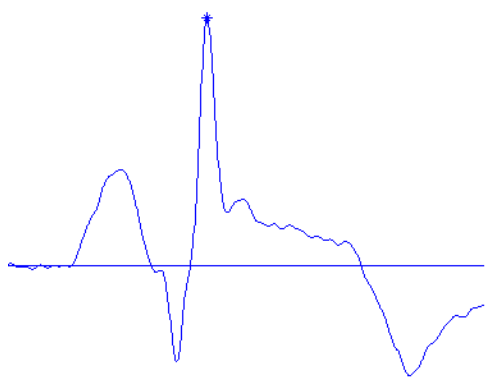

Fig 12: A sample where the $S$ point is not below the isoelectric line.

\subsection{Proposed algorithm for detection of ST elevation}

1. Find the S-point by determining the minimum value from previously located R-peak and $\mathrm{T}$ onset.

2. If S-point found below iso-electric line, traverse to the right and find the intersection point with the isoelectric line. Check for elevation from this intersection point till the $\mathrm{T}$ onset.

3. Else if the S-point is found above the iso-electric line then conclude the presence of ST elevation.

4. If the ECG graph fails to satisfy both the conditions, then ST elevation is absent.

\section{LOCALIZATION}

Once the ST elevation is detected, the localization is done on the basis of the presence of ST elevation in the leads. Each lead collects signals from a particular part of the heart. Based on the presence of ST elevation, the location of the affected part of the heart is predicted.

The heart consist of four chambers in which the blood flows, these chambers of the heart provide a multiple step pathway for blood to be first sent to the lungs for respiration. The upper chambers of the heart are known as the left and the right atria whereas the lower chambers are known as the left and the right ventricles. A wall of muscle known as the "septum" separates the left and the right atria and the left and the right ventricle.

Any blockage is a sign of the lack of blood supply to that part of the heart. This results in lack of oxygen to that part of the heart which ultimately leads to the deadening of tissues in that part. A lot of tissues being dead in a specific part of the heart is termed as "Myocardial Infarction".

For the correct identification of the deadening of the tissues in a specific part of the heart, the MI is labeled as per the region where the blockage occurs:

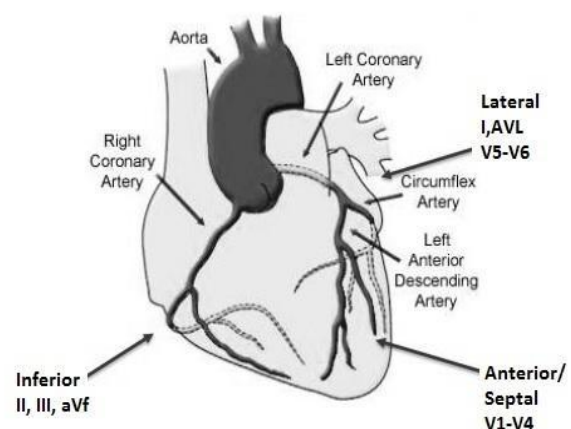

Figure 13: The representation of different regions of the heart and the associated leads.

- The anterior surface of the heart is formed mainly by the right atrium and the right ventricle. The right atrium and the right ventricle are separated by a Atrioventricular groove, where the right coronary artery is located. It is usually the front part of the heart.

- The posterior region (i.e. the base of the heart) is formed by the left atrium, into which the four pulmonary veins drain. This part is exactly opposite 
to the apex of the heart. This is the back portion of the heart.

- The region in between the two ventricles or the two atrias or an atria and a ventricle is known as the septum. This is the region where the arteries pass through.

- The lateral side of the heart is made by the left ventricle. The inferior surface is formed mainly by the right and left ventricle separated by the posterior interventricular groove. The inferior surface is also formed by the inferior part of the right atrium.

- The apex of the heart is formed by the left ventricle and is directed in such a way that it it is downward, forward and the left. This region is also known as the apical region.

According to the changes in these different zones as per the leads, the MI is localized. A combination of these regions is also used for localization.

The following table elaborates the identification of the MI as per the observation of the ST elevation in the leads:

Table 2: Significance of the ECG leads representing different regions of the heart [6].

\begin{tabular}{|c|c|c|c|}
\hline $\begin{array}{c}\text { LEAD I } \\
\text { Lateral }\end{array}$ & LEAD AVR & $\begin{array}{c}\text { LEAD V1 } \\
\text { Septal }\end{array}$ & $\begin{array}{c}\text { LEAD V4 } \\
\text { Anterior }\end{array}$ \\
\hline $\begin{array}{c}\text { LEAD II } \\
\text { Inferior }\end{array}$ & LEAD AVL & LEAD V2 & LEAD V5 \\
Leptal & Lateral \\
\hline $\begin{array}{c}\text { LEAD III } \\
\text { Inferior }\end{array}$ & LEAD AVF & LEAD V3 & LEAD V6 \\
Inferior & Anterior & Lateral \\
\hline
\end{tabular}

After once, the correct location of MI is detected we move further towards the identifying the culprit artery which has led to this blockage.

\section{CULPRIT ARTERY DETECTION}

The anatomy of the heart was studied with the help of the details mentioned in [7]. The heart is made up of different muscles. Like every other organ in the body, heart muscles also require oxygen rich blood supply for survival. The heart manages its blood supply with its own vascular system, known as "coronary circulation". The main supplier of the blood is the aorta which branches off into the left and right coronary arteries. The right coronary artery supplies blood to the right side of the heart. The right side of the heart is smaller since it pumps blood only to the lungs. The left side of the heart is much larger as compared to the right side since supplies blood to the rest of the body. Hence, the left coronary artery further branches into the left circumflex and left anterior descending artery.

Once ST segment detection is achieved in the previous modules, the use of a "culprit artery detection algorithm" [1] is proposed for further detection. Tierala proposed an algorithm for detecting RCA or LCx as the culprit artery. The original paper [1] is referred for studying and implementing the algorithm.

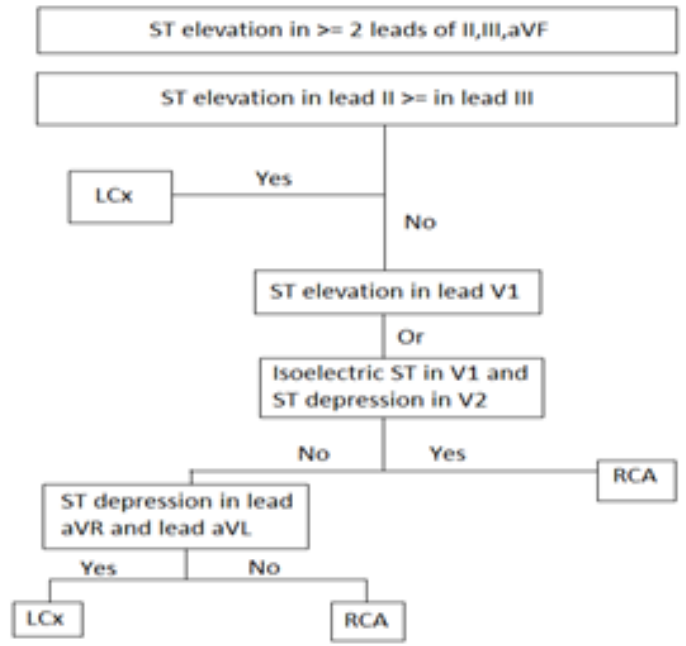

Figure 14: Algorithm of Tierala [1] for predictingRCA or LCX as the culprit artery.

\subsection{Algorithm for culprit artery detection}

[1]

1. Check for ST elevation in more than two leads of II, III and aVF.

2. If ST elevation in lead II is greater than or equal to in lead III, the culprit artery is LCx.

3. Else check for ST elevation in lead V1. If present, the culprit artery is RCA.

The proposed system concentrates on the detection of only ST elevated MI. Hence the cases with ST depression are not considered in the study.

\section{CONCLUSION}

A step-wise approach in automated ECG analysis has been proposed. Algorithms are applied which satisfy the requirements of the system. An algorithm which suits the needs of a particular module does not necessarily give you desired results, it is only after testing and analysis that you can determine which algorithm best fits into the systems' module. The algorithms like Gaussian filter, R-peak detection and isoelectric line detection were tested on a set of approximately 20 to 30 samples, and were found to be efficient which yielded the required results. These algorithms however require a prerequisite data to be assumed based on medical terminology before their implementation is undertaken.

The modules upto the iso-electric line detection have been successfully implemented using MATLAB. The images are the snapshots of actual implementation results. The succeeding modules in this paper are still under study. The design of these modules has been proposed based on the theoretical concepts.

An important aspect of the system is efficiency in analyzing risks to life. It allows people to identify the risk of MI. Its main advantage would be in areas where a specialist is not affordable. The proposed system does not eliminate the need for the specialist but acts as a support to the medical expert's opinion. 


\section{REFERENCES}

[1] NHJJ van der Putten, PR Rijnbeek, WA Dijk, G van Herpen, AC Maan, JA Lipton, JA Kors. Validation of ElectroCardiographic criteria for predicting the culprit artery in patients with acute myocardial infarction. Computing in Cardiology, 2010: 37:21-24.

[2] Algorithms for ECG feature Extraction: An Overview. Svenja Kutscher.Mälardalen University, School of Innovation, Design, and Technology.

[3] Elaine N Clark, Maria Sejersten, Peter Clemmensen, Peter W Macfarlane. Evaluating Enhancing the Acute Myocardial Infarction Criteria in the GlasgowElectrocardiogram Analysis Program by Including ST Depression. Computing in Cardiology, 2010;37:29-32.

[4] Seena V, Jerrin.Yomas. A Review on Feature Extraction and Denoising of Ecg Signal Using Wavelet Transform.
2014 2nd International Conference on Devices, Circuits and Systems (ICDCS)

[5] www.Physionet.orghttp://www.physionet.org/cgibin/atm/ATM.

[6] www.slideshare.nethttp://www.slideshare.net/mssa_500/ myocardial-ischemia-and-infarction. Presentation by Dr. MohmmedAL jaberi.

[7] Harrison's Principles of Internal Medicine, $17^{\text {th }}$ Edition.

[8] www.youtube.com https://www.youtube.com/watch?v=T_b9U5gn_Z

[9] http://en.ecgpedia.org/wiki/ST_Morphology

[10] Sunil Kumar Kopparapu, M Satish. Identifying optimal Gaussian Filter for Gaussian Noise Removal. 2011 Third National Conference on Computer Vision, Pattern Recognition, Image Processing and Graphics. 